\title{
BMJ Open Feasibility of repeated self-measurements of maximum step length and gait speed by community-dwelling older persons
}

\author{
Kim T J Bongers, Yvonne Schoon, Marcel G M Olde Rikkert
}

To cite: Bongers KTJ, Schoon Y, Olde Rikkert MGM. Feasibility of repeated self-measurements of maximum step length and gait speed by community-dwelling older persons. BMJ Open 2016;6: e011538. doi:10.1136/ bmjopen-2016-011538

- Prepublication history for this paper is available online. To view these files please visit the journal online (http://dx.doi.org/10.1136/ bmjopen-2016-011538).

Received 16 February 2016 Revised 13 May 2016 Accepted 26 May 2016

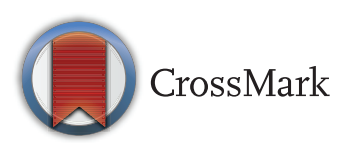

Department of Geriatric Medicine, Radboud University Medical Center, Radboud Institute for Health Sciences, Nijmegen, The Netherlands

Correspondence to Kim TJ Bongers; kim. bongers@radboudumc.nl

\section{ABSTRACT}

Objectives: Self-management of mobility and fall risk can be important in fall prevention; however, it remains unstudied. Therefore, the current study assessed whether community-dwelling older persons were able to repeatedly self-assess maximum step length (MSL) and gait speed (GS) in their own home for a 6-month period, how these tests changed during this period and if these changes were related to falling.

Design: This is a prospective study.

Setting: This study was conducted at home. Participants: A total of 56 community-dwelling older adults (24 women (43\%), mean age 76.2 (SD 3.9) years) entered the study; of which, 45 completed the study.

Methods: Participants performed MSL and GS once a week in their own home during a 6-month period.

Primary and secondary outcomes: Repeated MSL and GS measurements were the primary outcomes. Falls, self-management and mobility were the secondary outcomes.

Results: Self-assessment of MSL and GS by older persons is feasible. Compliance of repeatedly selfmeasuring MSL and GS was good; the median number of weekly measurements was $23.0(88 \%)$ and 21.0 $(81 \%)$ for MSL and GS, respectively. Drop-outs showed less self-management abilities compared to the participants who completed the study $(p=0.049)$. Linear mixed models showed a small significant improvement in MSL and GS over time $(p<0.001)$, without an influence on falling.

Conclusions: Most community-dwelling older persons are able and willing to repeatedly assess their MSL and GS. Self-managing mobility and fall risk did not increase fall occurrence. The fact that older persons can be actively involved in their own healthcare is clinically relevant. Further studies are needed to examine the (cost)effectiveness of self-management in fall prevention interventions.

\section{INTRODUCTION}

Falling is a major problem in today's ageing population, causing not only highly relevant physical and social impairments but also increasing health-related costs. ${ }^{1-3}$ Policymakers are strongly emphasising that older persons

\section{Strengths and limitations of this study}

- Prospective 6-month follow-up of repeated measurements of mobility and falls data.

- Measurements were performed by seniors themselves at their own home.

- The falls telephone system assesses falls during the study period reliable and valid. During the study period falls were reliably assessed using the falls telephone system.

- This study looks at the influence of falling on a group level, not on the preferable individual level.

- Participants were part of a prior study resulting in a possible selection bias.

should be more actively involved in their own healthcare, and scientists are following this lead on self-management, which is also underlined by the chronic care model. ${ }^{4-6}$ However, self-management of mobility and fall risk received little attention in previous research. Deteriorating mobility is a risk factor for falling, ${ }^{1}$ and the ability to quickly identify changes in their mobility could give older persons the opportunity to take responsibility of their own mobility-related well-being. Adequate self-management could minimise the impact of their fall tendency on their lives and could improve quality and effectiveness of our fall-related healthcare services.

Improving self-management of mobility and fall risk by identifying relevant changes in mobility is only possible with a simple and safe assessment tool that older persons can easily integrate in their normal daily life. Furthermore, such an assessment tool needs to be feasible, reliable, valid and should have the ability to sufficiently predict future falls. Previous studies showed the maximum step length (MSL) and usual gait speed (GS) to have the potential to be such self-assessment tools. ${ }^{2}$ 7-19

MSL is a simple and reliable measure for the assessment of balance and mobility in 
community-dwelling and frail older individuals. ${ }^{2}$ 7-10 Predictive studies linking MSL to fall risk are still limited. ${ }^{911}$ GS proved to be a feasible and valid measure to predict future adverse events, such as disability and falls, in community-dwelling older persons. ${ }^{11-19}$ An important limitation of most of these studies was that only a single-baseline MSL or GS measurement was assessed by a professional in a clinical setting. It can be reasoned that mobility assessment at only one time point may preclude identification of a large proportion of the persons at risk as they may experience a rapid or gradual decline in mobility and balance over time, and may or may not pass in due time a population-based cut-off point. ${ }^{19}$ Also, single or infrequent evaluations may not be representative of a person's true locomotion ability, because of large within-participant variability in frail older persons. ${ }^{19-21}$

In our previous research, we already showed that MSL and GS are safe, feasible and reliable self-assessment tools of mobility and fall risk for community-dwelling older persons. ${ }^{22}$ However, research on repeatedly measuring MSL and GS over a longer period of time is still lacking. Therefore, the current exploratory study primarily investigated the feasibility of repeatedly measuring MSL and GS by community-dwelling older persons for a 6-month period, but also how MSL and GS changed during this period, whether these changes were related to experiencing a fall and what the implications of these results are in the field of self-management research in older persons.

\section{METHODS}

\section{Participants}

This prospective study was a preplanned part of the Validation Study of the Two-step Older persons Screening study (TOS study), in which six general practitioners' practices organised frailty screening for all their patients aged 70 years and over. ${ }^{23}$ Detailed information about the rational and design of the TOS study, and the subsequent recruitment of the participants for the Senior Step Study were described previously. ${ }^{22} 23$ Participants and their informal caregivers from two participating practices outside the city of Nijmegen, the Netherlands, were asked to participate in the Senior Step Study. Individuals were excluded when they were not able to understand the instructions to perform the tests, not able to walk (with or without a walking aid), did not speak and understand Dutch, were not able to answer the falls telephone (FT) or did not have an informal caregiver who could answer the FT for them. All participants provided written informed consent. The research ethics committee-region Arnhem-Nijmegenapproved the study (approval number 2009/223).

\section{Design}

A 6-month follow-up period was chosen to explore the repeated assessments of MSL and GS by older persons. Participants were advised to perform the MSL and GS at home once a week, on the same day and around the same time, accompanied by their informal caregivers. The informal caregiver was asked to record the time needed to perform GS, to avoid dual tasking of the participant. The researcher explained and practiced the potential self-tests with the participants and their informal caregivers during the first study visit, which took place at the participant's home. Instructions were given a maximum of three times, and the researcher judged whether the participant and caregiver were able to execute the self-tests correctly and safely. If the participants and/or caregivers were not able to perform the self-tests independently, the participant was excluded. ${ }^{22}$ After 1 month, the researcher revisited the participant and their informal caregiver and asked them to execute the self-tests. Errors made were recorded by the researcher and explained to the participant. At the end of the study, the researcher visited the participant again and recorded errors made during the performance of the self-tests.

\section{Descriptive measures}

Mobility was assessed by the researcher at the participant's home at baseline and monthly during the 6-month follow-up using the standardised balance and mobility measures: Timed Up and Go (TUG) ${ }^{24} 25$ and Short Physical Performance Battery (SPPB) ${ }^{26}$ For TUG, the participant, when seated with their back against a chair, was instructed to stand up, walk $3 \mathrm{~m}$ past a mark on the floor as quickly as possible, turn around, walk back to the chair and sit down again with their back against the chair. ${ }^{24}{ }^{25}$ The time in seconds needed to complete this test was measured. TUG was performed twice, and the fastest performance was registered. The SPPB included GS (time to walk $4 \mathrm{~m}$ at their normal walking speed), five chair stands (time to rise from a chair and return to a seated position without using arms) and a balance test (ability to stand with the feet together in the side-by-side, semitandem and tandem positions); all measured using a stopwatch. A summary performance score (range 0-12) for the three tasks was created for each participant, higher scores indicating better lower body function. ${ }^{26}$

At baseline and after 6 months, the following questionnaires were assessed: disability was evaluated with the (modified) Katz scale (Katz-15 item scale, which measures basic and instrumental activities of daily living, with a score range of $0-15$, lower scores indicating a better functional performance) $;^{27}$ disease burden was assessed with the Cumulative Illness Rating Scale for Geriatrics (CIRS-G; scored on a scale from 0 to 4 for each of 14 categories, higher scores reflecting more comorbidity) $;^{28}$ the Self-Management Ability Scale (SMAS-30) was used to evaluate self-management (30 items, score range $0-100$, higher scores indicating better self-management) $;^{29}$ and daily physical activity was assessed using the LASA Physical Activity Questionnaire (LAPAQ), which determined walking, cycling, 
gardening, sports and household activities (scoring the total minutes per day for all physical activities). ${ }^{30}$

\section{Falls}

A fall was defined as 'an unexpected event in which the subject comes to rest on the ground, floor, or lower level'. ${ }^{31}$ Fall incidents were monitored by the FT during the 6-month follow-up. The FT system (ASK Community Systems, Rotterdam, the Netherlands) is a computerised system that automatically contacts participants by telephone using pre-recorded messages and was found to be a feasible, reliable and valid method of assessing falls in older people. ${ }^{32} 33$ Participants were automatically phoned by the FT system once a week on their day of preference, and participants were asked to report the number of falls in the past week twice (in case a wrong number was entered the first time). The system called back up to a maximum of four times a day and tried again the following day if the call was not answered. The research assistant called participants to verify each registered fall and participants with no reply to the FT.

\section{Self-tests}

Maximum step length

Participants measured their MSL without the help of their informal caregiver. The present study used the modified version of MSL first described by Schoon et $a l^{10}$ Participants were provided with a poster displaying a ruler in centimetres and with an indication for the initial position of the feet $(170 \times 50 \mathrm{~cm}$, made by the researchers). To prevent participants from slipping during the performance of the test, antislip was attached to the underside of the poster. Participants were instructed to stand with both feet on the indicated position and to step maximally forward on the printed ruler with their right leg, and subsequently bring the left leg up to the first leg in one step. In this position, participants could read their distance stepped. When the participant stepped in one fluid movement, a trial was successful, and when more than one step was needed to maintain balance or when balance was lost, a trial was unsuccessful. Every week, participants performed two practice trials after which they performed the MSL at least three times, with a maximum of five trials to obtain three successful trials. Every week, participants wore the same firm comfortable shoes, which were selected by the researcher during the first study visit.

\section{Gait speed (GS)}

Participants measured their GS with help of their informal caregiver. A track between 5.5 and $6.5 \mathrm{~m}$ at the participant's home, where the participant had to start and finish the walking track, was indicated by the researcher during the first study visit. The informal caregiver was instructed to measure the time needed to cover the distance between two fixed landmarks along this track (eg, furniture, door posts, paintings), and to start and stop the time, respectively. Depending on the furniture in the participant's home, the distance between the two landmarks was between 3.5 and $4.5 \mathrm{~m}$, and this distance was used for the analyses. Participants performed GS twice at their normal walking speed and wore the same shoes as during MSL.

\section{Analyses}

Leg length, measured during the TOS study as the distance between the anterior superior iliac spine and lateral malleolus, was used to normalise MSL by dividing step length by leg length. The maximum MSL among the three successful step lengths was used as the MSL of that specific week. Distance was divided by time in $\mathrm{m} / \mathrm{s}$ to calculate GS, and the mean of the two walks of that specific week was used in the analysis.

\section{Feasibility}

Reasons for drop-out were registered, and descriptive variables assessed at baseline of the study of the persons who dropped out were compared to those who completed the study using independent-sample t-tests for continuous variables and $\chi^{2}$ tests for categorical variables. The same analyses were used to compare participants who fell during the 6-month follow-up (fallers) to those who did not fall (non-fallers).

A full description of the errors in self-testing mobility seen by the researchers was described previously. ${ }^{22}$ Feasibility of repeatedly measuring MSL and GS was explored by looking at the median and quartiles of the number of weekly measurements. This was performed for all participants together and also for the participants who completed the study. A coefficient of variation $(\mathrm{CV}$ : $\mathrm{SD}_{\text {repeated measurements }} /$ mean $_{\text {of all assessments so far }}$ ) over all weekly measurements was calculated for each participant separately to demonstrate the variability of the self-tests within each participant.

\section{Analysis of changes over time}

Differences between the measurements of weeks 1 and 26 were calculated. Since not all participants had a measurement in weeks 1 and 26, differences were also calculated between the first and last measurements of each participant. The course of MSL and GS over time (each week during the 6-month period) was explored using error bars displaying means and 95\% CIs. Linear mixed models with random effects were used to study all weekly measurements of MSL and GS. Having a fall history and experiencing a fall during the 6-month follow-up were added as covariates. Changes in TUG, SPPB, Katz-15, CIRS-G, SMAS-30 and LAPAQ between baseline and after 6 months were compared using paired samples t-tests.

Significance was set at $\mathrm{p}<0.05$. All statistical analyses were performed using IBM SPSS Statistics V.20 (SPSS, Chicago, Illinois, USA). 
Table 1 Baseline characteristics of the senior step study population, the participants who completed this study and the drop-outs

\begin{tabular}{lcccl}
\hline & Study population $\mathbf{( N = 5 6 )}$ & Participants $(\mathbf{N}=\mathbf{4 5})$ & Drop-outs $(\mathbf{N}=\mathbf{1 1})$ & p Value \\
\hline Age, years & $76.2(3.9)$ & $75.8(3.9)$ & $77.7(3.5)$ & 0.154 \\
Female, N (\%) & $24(42.9)$ & $19(42.2)$ & $5(45.5)$ & 0.554 \\
SPPB & $10.6(1.7)$ & $10.7(1.5)$ & $10.0(2.3)$ & 0.230 \\
TUG, S & $7.9(2.5)$ & $7.6(1.8)$ & $9.0(4.3)$ & 0.336 \\
LAPAQ, min/day & $151.3(88.6)$ & $156.1(84.9)$ & $129.5(106.0)$ & 0.396 \\
SMAS-30 & $64.7(12.7)$ & $66.3(13.1)$ & $57.6(8.1)$ & $0.049^{*}$ \\
Katz-15 & $0.7(1.0)$ & $0.6(0.9)$ & $0.9(1.3)$ & 0.440 \\
CIRS-G & $8.1(4.4)$ & $7.8(4.5)$ & $9.5(3.7)$ & 0.250 \\
Fall history, N (\%) & $21(37.5)$ & $15(33.3)$ & $6(54.5)$ & 0.169 \\
\hline
\end{tabular}

All data presented as means \pm SD unless stated otherwise.

*p Value $<0.05$

CIRS-G, Cumulative IIIness Rating Scale for Geriatrics, scored on a scale from 0 to 4 for each of the 14 categories, higher scores reflecting more comorbidity; Katz-15, Katz-15 item scale, basic and instrumental activities of daily living, with a score range of 0-15, lower scores indicating better functional performance; LAPAQ, LASA Physical Activity Questionnaire, minutes per day for sports activities, non-sports activities and all physical activities; SMAS-30, Self-Management Ability Scale, 30 items, score range 0-100, higher scores indicating better self-management; SPPB, Short Physical Performance Battery, summary performance score (range 0-12) for three tasks, higher scores indicating better lower body function; TUG, Timed Up and Go.

\section{RESULTS}

Fifty-six older adults with a mean age of 76.2 years (SD $3.9)$, with 24 women $(42.9 \%)$ and their informal caregivers consented to participate in the Senior Step Study. ${ }^{22}$

\section{Feasibility}

In total 11 out of the 56 participants (19.6\%) dropped out of the Senior Step Study. Seven of which dropped out within the first month and gave the following reasons: two dropped out because of a death in the family, three had other expectations of the study, one was diagnosed with dementia and not able to understand the instructions and one developed a tendonitis of the knee. One participant dropped out after 3 months because it was physically too demanding and her informal caregiver was not able to perform the measurements correctly. Finally, three participants dropped out during the last month of the study: one experienced a fall not related to the study and suffered a hip fracture, one was diagnosed with colon cancer and had to be operated on immediately, and one suffered from encephalitis which resulted in a hospital and nursing home stay. Drop-outs differed significantly at baseline from the participants who completed the study in that they showed less self-management ability ( $\mathrm{p}=0.049$, table 1$)$. Participants who fell during the 6-month follow-up did not differ significantly from those who did not fall. Fifteen participants fell once during the follow-up, 1 participant fell twice, 1 participant fell three times and 2 participants each fell four times.

In the study group (including drop-outs), the feasibility of repeatedly self-measuring MSL and GS during the 26-week period was high with a median $(25 \%$ and $75 \%$ centiles) number of weekly measurements of 23.0 (18.0 and 25.8) and 21.0 (16.3 and 23.8) for MSL and GS, respectively. When only looking at the participants who completed the study $(n=45)$, feasibility was even higher for MSL and GS (median of 24.0 (21.0 and 26.0) and median of 22.0 (20.0 and 24.0), respectively). Almost all participants without any weekly measurements were the drop-outs from within the first month. The exception was the drop-out from within the third month of follow-up.

Intraindividual variation calculated as the $\mathrm{CV}$ per participant for both self-tests was small (median of $3.5 \%$ for MSL and $6.5 \%$ for GS; figure 1 ).

\section{Changes over time}

Mean $( \pm \mathrm{SD})$ difference between weeks 1 and 26 in the study group was $0.09(0.10)$ and $0.10(0.13)$ for MSL and GS, respectively, while the mean $( \pm \mathrm{SD})$ difference

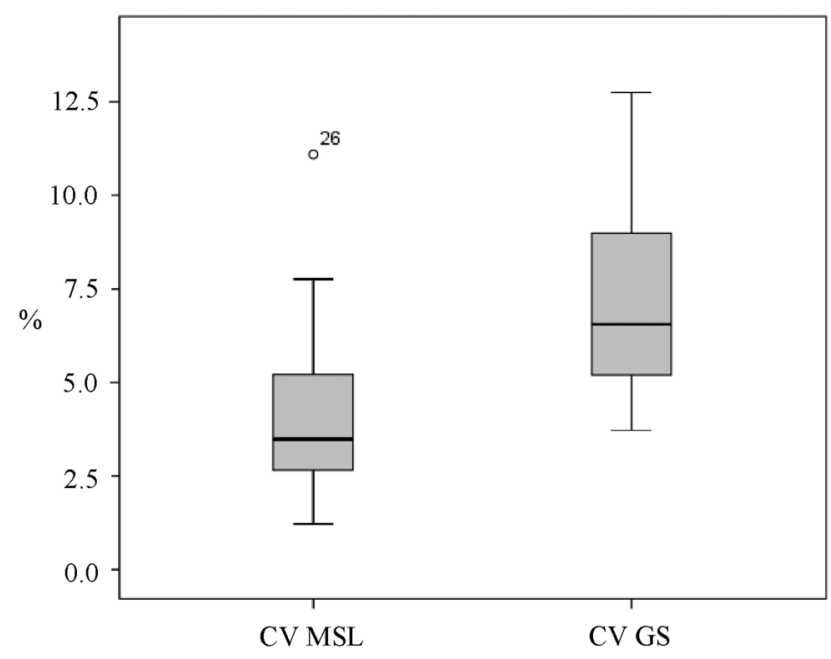

Figure 1 Boxplots of the intraindividual coefficients of variation in a 26 -week time series for each self-test. Boxplots display median and quartiles. CV, coefficient of variation; MSL, maximum step length; GS, gait speed. 

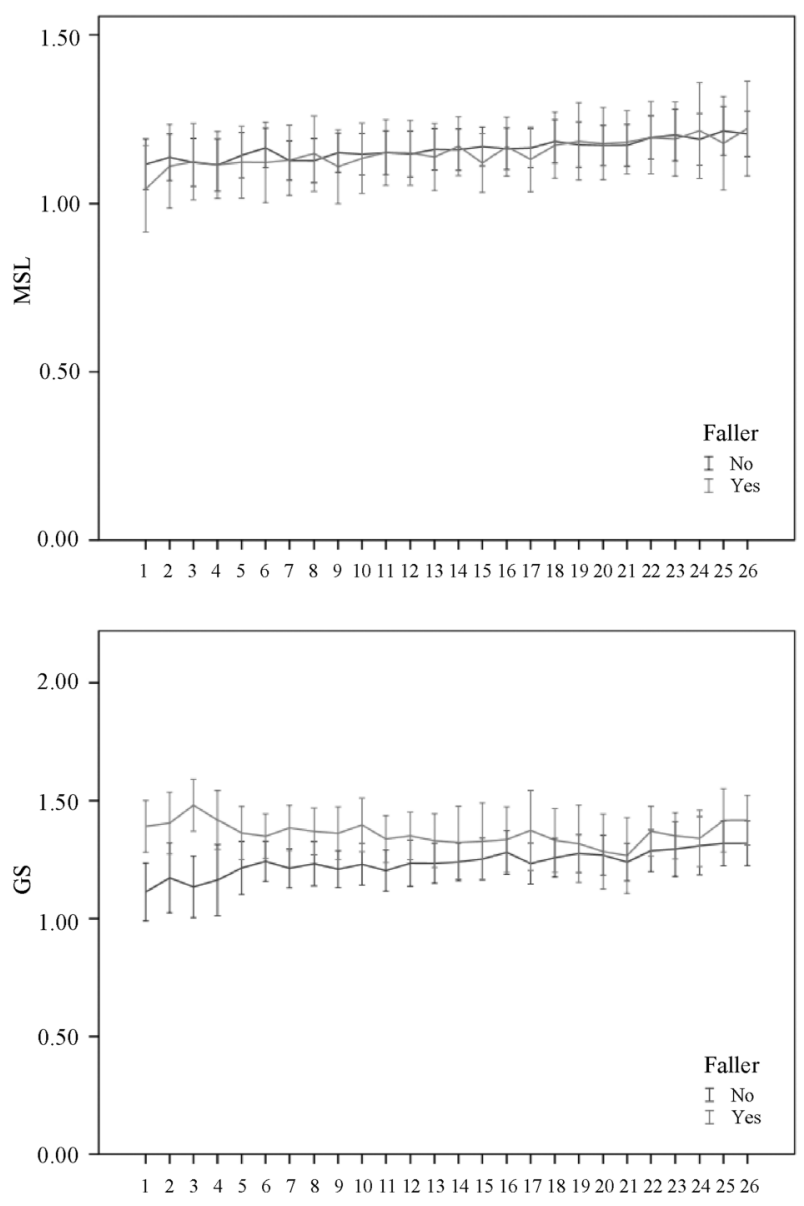

Figure 2 Mean and $95 \%$ Cls of the course over the 6-month follow-up of the MSL and GS measured by seniors who fell at least once during the follow-up period compared with non-fallers. MSL, maximum step length; GS, gait speed.

between the first and last measurements was $0.09(0.09)$ and $0.07(0.13)$ for MSL and GS, respectively. The course of MSL and GS over the 6-month follow-up period is depicted in figure 2. Linear mixed models with random effects showed a significant but small improvement in MSL and GS over time $(\mathrm{p}<0.001$; table 2$)$. Having a fall history or experiencing a fall during the follow-up did not have a significant effect on this improvement. The mobility measures TUG, and SPPB improved significantly between baseline and after 6 months (7.59 vs $7.14 \mathrm{~s}(\mathrm{p}=0.004)$ and 10.69 vs 11.24 ( $\mathrm{p}=0.018$ ) for TUG and SPPB, respectively).

\section{DISCUSSION}

The main finding of this study was the high number of weekly measurements and the small intraindividual variation which showed that older people are willing and able to repeatedly assess their MSL and GS in their own homes for a period of 6 months as a measure of their mobility. Repeatedly self-assessing MSL and GS resulted in a slight improvement on these measures, which was mirrored by the improved mobility measured by TUG and SPPB, but did not result in improved self- management ability scores. Having a history of falling or experiencing a fall during follow-up did not influence these results.

To the author's knowledge, this was the first study to explore whether older persons are able to repeatedly measure their MSL and GS at home as a measure of their mobility and fall risk. The good compliance of more than $80 \%$ in this study confirmed this hypothesis and therefore showed that it is possible to get older people to be actively involved in the management of their own mobility and fall risk, which is in line with studies showing the possibility of self-management in other chronic diseases, such as chronic obstructive pulmonary disease and diabetes. ${ }^{34} 35$ However, the small improvement on the self-tests and mobility measures over time does give concern about the reliability of monitoring with these tests. Obviously, the weekly assessments of MSL and GS seem to train older persons, and it would, therefore, be very interesting to study whether this improvement lasts during a longer follow-up period. This may limit the validity of these tests as a screening tool for fall risk (in case this does not change), but on the other hand, it may mean a possible therapeutic effect of repeatedly performing MSL and GS by community-dwelling older persons. We did not find an improvement in ADL activities and total activity minutes per day as measured by the LAPAQ, so the effect of these tests as training tools needs to be further studied.

Although the study population measured their MSL and GS very regularly, no change in self-management abilities was found. SMAS-30 was already validated and proved to be reliable in an older population, with good reproducibility after 16 weeks. $^{29}$ It could be reasoned that the 6-month follow-up was too short to achieve improvements in self-management abilities. The SMAS-30 scores were comparable to the scores of community-dwelling older and partly frail individuals found in the studies of Schuurmans et $a l^{29}$ but higher compared to a study with 55-year-old lonely women. ${ }^{36}$ Since the drop-outs in the current study showed less selfmanagement abilities compared to the participants who completed the study, it could be reasoned that the study population already consisted of a selection of selfmanaging older persons, which limits the feasibility of our self-tests for a frailer population.

\section{Strengths and limitations}

A strength of this Senior Step Study was the prospective follow-up of many descriptives over 6 months. Furthermore, the $19.6 \%$ drop-out rate was comparable to other studies with frail older persons and can be interpreted as rather low as it demanded a higher responsibility of the participants included compared with other studies in which treatments are simply administered.

A limitation of this study was the possible selection bias. $^{22}$ Compared to the non-participating individuals, the Senior Step Study population did show a good 
Table 2 Linear mixed models with random effects showing the change of MSL and GS over time and the possible influence of being a faller or having a fall history on this change

\begin{tabular}{|c|c|c|c|c|}
\hline & & B & p Value & $95 \% \mathrm{Cl}$ \\
\hline \multirow[t]{3}{*}{$\mathrm{MSL}^{*}$} & Intercept & 1.113 & $<0.001$ & 1.066 to 1.161 \\
\hline & Time & 0.004 & $<0.001$ & 0.003 to 0.005 \\
\hline & Residual & 0.001 & $<0.001$ & 0.001 to 0.001 \\
\hline \multirow[t]{5}{*}{ MSL† } & Intercept & 1.129 & $<0.001$ & 1.072 to 1.186 \\
\hline & Time & 0.003 & $<0.001$ & 0.002 to 0.005 \\
\hline & Fall history & -0.048 & 0.342 & -0.148 to 0.052 \\
\hline & Timexfall history & 0.001 & 0.570 & -0.002 to 0.003 \\
\hline & Residual & 0.001 & $<0.001$ & 0.001 to 0.001 \\
\hline \multirow[t]{5}{*}{ MSL $\ddagger$} & Intercept & 1.125 & $<0.001$ & 1.066 to 1.184 \\
\hline & Time & 0.003 & $<0.001$ & 0.002 to 0.004 \\
\hline & Faller & -0.032 & 0.516 & -0.129 to 0.066 \\
\hline & Timexfaller & 0.001 & 0.305 & -0.001 to 0.003 \\
\hline & Residual & 0.001 & $<0.001$ & 0.001 to 0.001 \\
\hline \multirow[t]{3}{*}{$\mathrm{GS}^{*}$} & Intercept & 1.240 & $<0.001$ & 1.170 to 1.311 \\
\hline & Time & 0.002 & 0.022 & 0.0003 to 0.004 \\
\hline & Residual & 0.007 & $<0.001$ & 0.006 to 0.008 \\
\hline \multirow[t]{5}{*}{ GS† } & Intercept & 1.222 & $<0.001$ & 1.136 to 1.307 \\
\hline & Time & 0.002 & 0.023 & 0.000 to 0.004 \\
\hline & Fall history & 0.056 & 0.446 & -0.091 to 0.204 \\
\hline & Timexfall history & -0.001 & 0.452 & -0.005 to 0.002 \\
\hline & Residual & 0.007 & $<0.001$ & 0.006 to 0.008 \\
\hline \multirow[t]{5}{*}{ GS $\ddagger$} & Intercept & 1.197 & $<0.001$ & 1.112 to 1.283 \\
\hline & Time & 0.003 & 0.011 & 0.001 to 0.005 \\
\hline & Faller & 0.116 & 0.103 & -0.024 to 0.256 \\
\hline & Timexfaller & -0.002 & 0.204 & -0.006 to 0.001 \\
\hline & Residual & 0.007 & $<0.001$ & 0.006 to 0.008 \\
\hline
\end{tabular}

${ }^{*}$ Model including only the self-test (MSL or GS).

†Model including the self-test (MSL or GS), time and having a fall history.

¥Model including the self-test (MSL or GS), time and having experienced a fall during the 6-month follow-up.B, estimate; GS, gait speed; MSL, maximum step length.

overall performance in mobility making it difficult to generalise the observed ability of repeatedly self-assessing MSL and GS to a more frailer population. When looking at falling and self-management abilities, the selection bias seemed limited in this study population. Fall prevalence was comparable to a community-dwelling older population $(38.5 \%),{ }^{37}$ the TOS study $(35 \%$ had a fall history) ${ }^{38}$ and the follow-up study (36\%). As mentioned above, self-management abilities were comparable to those found by Schuurmans $e t a l,{ }^{29}$ but higher compared with the findings of Kremers $e t a l^{36}$ Another limitation was that the current study only looked at the influence of falling on a group level. Since there were only 19 fallers, the sample size was too small to accurately explore the relation between repeated measurements of MSL and GS and falls in individual cases and whether changes in the self-tests within individuals could be predictive for future falls or other negative health outcomes. Therefore, it would be interesting to repeat the study in a larger and frailer population.

\section{CONCLUSIONS}

The implications for clinical practice and future research are promising. The good compliance shows that we can engage older persons in their own healthcare. This may also open possibilities for self-management in other healthcare areas, such as frailty and functional status. MSL and GS are simple one-item tools, and perhaps other one-item tools for other health conditions can also be performed by seniors themselves. The definitive place of the MSL and GS in prevention and monitoring falls according to the chronic care model requires further study, including self-management. It is promising that self-management of mobility and fall risk did not increase falling.

If we want to engage older persons to take an active part in their own healthcare, especially in fall prevention, interventions should be shaped in a way that suites them. This Senior Step Study took the first step by exploring that older persons were willing and able to use MSL and GS weekly as a self-management tool for monitoring their mobility and fall risk. Further studies are needed to examine the exact relation of these selfmanagement capabilities with fall prevention and to confirm that such self-management capabilities can also be realised in larger and frailer populations.

Acknowledgements The researchers thank Janneke van Kempen and René Melis for allowing the Senior Step Study to be part of the TOS study. They also thank Maartje Graauwmans for her contribution to the data acquisition. 
Contributors KTJB involved in execution of the study, supervision and acquisition of participant measurements, analysis and interpretation of the data, literature review and drafted the manuscript. YS contributed to the design of the study, involved in interpretation of the data and critically reviewed the manuscript. MGMOR contributed to the design of the study, supervised the study, involved in interpretation of the data and critically reviewed the manuscript

Funding This study was supported by the National Programme for Elderly Care (grant number 314050301), which is coordinated and sponsored by ZonMw, the Netherlands, Organisation of Health Research and Development.

Competing interests All authors had financial support from ZonMw, the Netherlands, Organisation of Health Research and Development, for the submitted work; no financial relationships with any organisations that might have an interest in the submitted work in the previous 3 years; and no other relationships or activities that could appear to have influenced the submitted work.

Ethics approval Ethics approval was obtained from research ethics committee-region Arnhem-Nijmegen.

Provenance and peer review Not commissioned; externally peer reviewed.

Open Access This is an Open Access article distributed in accordance with the Creative Commons Attribution Non Commercial (CC BY-NC 4.0) license, which permits others to distribute, remix, adapt, build upon this work noncommercially, and license their derivative works on different terms, provided the original work is properly cited and the use is non-commercial. See: http:// creativecommons.org/licenses/by-nc/4.0/

\section{REFERENCES}

1. Robinovitch SN, Feldman F, Yang Y, et al. Video capture of the circumstances of falls in elderly people residing in long-term care: an observational study. Lancet 2013;381:47-54.

2. Medell JL, Alexander NB. A clinical measure of maximal and rapid stepping in older women. J Gerontol A Biol Sci Med Sci 2000;55: M429-33.

3. Bertera EM, Bertera RL. Fear of falling and activity avoidance in a national sample of older adults in the United States. Health Soc Work 2008;33:54-62.

4. Barlow J, Wright C, Sheasby J, et al. Self-management approaches for people with chronic conditions: a review. Patient Educ Couns 2002;48:177-87.

5. Frieswijk N, Steverink N, Buunk BP, et al. The effectiveness of a bibliotherapy in increasing the self-management ability of slightly to moderately frail older people. Patient Educ Couns 2006;61:219-27.

6. Wagner EH, Austin BT, Davis C, et al. Improving chronic illness care: translating evidence into action. Health Aff (Millwood) 2001;20:64-78

7. Cho BL, Scarpace D, Alexander NB. Tests of stepping as indicators of mobility, balance, and fall risk in balance-impaired older adults. $J$ Am Geriatr Soc 2004:52:1168-73.

8. Goldberg A, Schepens S, Wallace M. Concurrent validity and reliability of the maximum step length test in older adults. J Geriatr Phys Ther 2010;33:122-7.

9. Lindemann U, Lundin-Olsson L, Hauer K, et al. Maximum step length as a potential screening tool for falls in non-disabled older adults living in the community. Aging Clin Exp Res 2008;20:394-9.

10. Schoon $Y$, Weerdesteyn V, Stunnenberg A, et al. Sense and simplicity: maximum step length is also reliable, feasible, and valid in very old adults. J Am Geriatr Soc 2010;58:2444-5.

11. Bongers KT, Schoon Y, Graauwmans MJ, et al. The predictive value of gait speed and maximum step length for falling in community-dwelling older persons. Age Ageing 2015;44:294-9.

12. Abellan van Kan $G$, Rolland $Y$, Andrieu $S$, et al. Gait speed at usual pace as a predictor of adverse outcomes in community-dwelling older people an International Academy on Nutrition and Aging (IANA) Task Force. J Nutr Health Aging 2009;13:881-9.

13. Studenski S, Perera S, Patel K, et al. Gait speed and survival in older adults. JAMA 2011;305:50-8.

14. Viccaro LJ, Perera S, Studenski SA. Is timed up and go better than gait speed in predicting health, function, and falls in older adults? J Am Geriatr Soc 2011:59:887-92.

15. Chu LW, Chiu AY, Chi I. Impact of falls on the balance, gait, and activities of daily living functioning in community-dwelling Chinese older adults. J Gerontol A Biol Sci Med Sci 2006;61:399-404.
16. Montero-Odasso M, Schapira M, Soriano ER, et al. Gait velocity as a single predictor of adverse events in healthy seniors aged 75 years and older. J Gerontol A Biol Sci Med Sci 2005;60:1304-9.

17. Lamb SE, McCabe C, Becker C, et al. The optimal sequence and selection of screening test items to predict fall risk in older disabled women: the Women's Health and Aging Study. J Gerontol A Biol Sci Med Sci 2008;63:1082-8.

18. Paul SS, Canning CG, Sherrington C, et al. Three simple clinical tests to accurately predict falls in people with Parkinson's disease. Mov Disord 2013;28:655-62.

19. Scott D, McLaughlin P, Nicholson GC, et al. Changes in gait performance over several years are associated with recurrent falls status in community-dwelling older women at high risk of fracture. Age Ageing 2015;44:287-93.

20. Stone E, Skubic M, Rantz M, et al. Average in-home gait speed: investigation of a new metric for mobility and fall risk assessment of elders. Gait Posture 2015;41:57-62.

21. Kaye J, Mattek N, Dodge $\mathrm{H}$, et al. One walk a year to 1000 within a year: continuous in-home unobtrusive gait assessment of older adults. Gait Posture 2012;35:197-202.

22. Bongers KT, Schoon Y, Graauwmans MJ, et al. Safety, feasibility and reliability of the maximal step length, gait speed and chair test measured by seniors themselves: the Senior Step study. J Aging Phys Act 2015;23:438-43.

23. van Kempen JA, Schers HJ, Jacobs A, et al. Development of an instrument for the identification of frail elderly as target-population for intergrated care. Br J Gen Pract 2013;63:e225-31.

24. Mathias S, Nayak US, Isaacs B. Balance in elderly patients: the "get-up and go" test. Arch Phys Med Rehab 1986;67:387-9.

25. Podsiadlo D, Richardson S. The timed "up \& go": a test of basic functional mobility for frail elderly persons. J Am Geriatr Soc 1991;39:142-8.

26. Guralnik JM, Simonsick EM, Ferrucci L, et al. A short physical performance battery assessing lower extremity function: association with self-reported disability and prediction of mortality and nursing home admission. J Gerontol 1994;49:M85-94.

27. Weinberger M, Samsa GP, Schmader K, et al. Comparing proxy and patients' perceptions of patients' functional status: results from an outpatient geriatric clinic. J Am Geriatr Soc 1992;40:585-8.

28. Miller MD, Paradis CF, Houck PR, et al. Rating chronic medical illness burden in geropsychiatric practice and research: application of the Cumulative Illness Rating Scale. Psychiatry Res 1992;41:237-48.

29. Schuurmans $H$, Steverink $N$, Frieswijk $N$, et al. How to measure self-management abilities in older people by self-report. The development of the SMAS-30. Qual Life Res 2005;14: 2215-28.

30. Stel VS, Smit JH, Pluijm SM, et al. Comparison of the LASA Physical Activity Questionnaire with a 7-day diary and pedometer. $J$ Clin Epidemiol 2004;57:252-8.

31. Lamb SE, Jørstad-Stein EC, Hauer K, et al., Prevention of Falls Network Europe and Outcomes Consensus Group. Development of a common outcome data set for fall injury prevention trials: the prevention of Falls Network Europe consensus. J Am Geriatr Soc 2005;53:1618-22.

32. van der Marck MA, Overeem S, Klok PC, et al. Evaluation of the falls telephone: an automated system for enduring assessment of falls. J Am Geriatr Soc 2011;59:340-4.

33. Reelick MF, Faes MC, Lenferink A, et al. The fall telephone for falls assessment in frail older persons; feasibility, reliability, and validity. J Am Geriatr Soc 2011;59:372-3.

34. Barlow JH, Sturt J, Hearnshaw H. Self-management interventions for people with chronic conditions in primary care: examples from arthritis, asthma and diabetes. Health Educ J 2002;61: 365-78.

35. Kaptein AA, Fischer MJ, Scharloo M. Self-management in patients with COPD: theoretical context, content, outcomes, and integration into clinical care. Int J Chron Obstruct Pulmon Dis 2014:9:907-17.

36. Kremers IP, Steverink N, Albersnagel FA, et al. Improved self-management ability and well-being in older women after a short group intervention. Aging Ment Health 2006;10:476-84.

37. Hausdorff JM, Rios DA, Edelberg HK. Gait variability and fall risk in community-living older adults: a 1-year prospective study. Arch Phys Med Rehabil 2001;82:1050-6.

38. Schoon $\mathrm{Y}$, Bongers $\mathrm{K}$, Van Kempen J, et al. Gait speed as a test for monitoring frailty in community-dwelling older people has the highest diagnostic value compared to step length and chair rise time. Eur J Phys Rehabil Med 2014;50:693-701. 